\title{
Temperature-dependence of polymer grating couplers for single-mode waveguides
}

Pei Li, Stanislav Sherman, Maher Rezem, Kirsten Honnef, Hans Zappe

Pei Li, Stanislav Sherman, Maher Rezem, Kirsten Honnef, Hans Zappe, "Temperature-dependence of polymer grating couplers for single-mode waveguides," Proc. SPIE 10535, Integrated Optics: Devices, Materials, and Technologies XXII, 105351R (23 February 2018); doi: 10.1117/12.2289444 


\title{
Temperature-dependence of polymer grating couplers for single mode waveguides
}

\author{
Pei $\mathrm{Li}^{*}$ a , Stanislav Sherman ${ }^{\mathrm{a}}$, Maher Rezem ${ }^{\mathrm{b}}$, Kirsten Honnef ${ }^{\mathrm{c}}$, and Hans Zappe ${ }^{\mathrm{a}}$ \\ ${ }^{a}$ Gisela and Erwin Sick Chair of Micro-optics, Department of Microsystems Engineering - \\ IMTEK, University of Freiburg, Georges-Koehler-Allee 102, Freiburg, Germany \\ ${ }^{\mathrm{b}}$ Hannover Centre for Optical Technologies (HOT), Leibniz University Hannover, Hannover, \\ Germany \\ ${ }^{\mathrm{c}}$ Laboratory for Materials Processing, Department of Microsystems Engineering - IMTEK, \\ University of Freiburg, Georges-Koehler-Allee 102, Freiburg, Germany
}

\begin{abstract}
Polymer optical sensors have attracted significant scientific interest due to the advantages of flexibility and lowcost mass-production possibilities. A main challenge in integration of all-polymer systems is interfacing sensor parts to light sources and detectors. Coupling strategies such as end-facet coupling, $45^{\circ}$ mirrors coupler and gratings coupler have been investigated. However, facet polishing is complicated for large flexible foils, while $45^{\circ}$ mirrors often require a metal reflection layer. Thus grating couplers have the biggest potential and are compatible with roll-to-roll processes. Polymers have higher thermo-optic-coefficients and thermal-expansioncoefficients compared to inorganic materials, making them very sensitive to temperature changes. Consequently, polymer grating couplers show a shift of the coupling angle with temperature and thus a decay of efficiency. We therefore present a temperature characterization of all-polymer based grating couplers on waveguides based sensors. Cost-effective manufacturing methods, including hot-embossing and spin-coating, were used for the fabrication of waveguides and gratings on PMMA foils. Single mode waveguides were realized by modifying the dimensions of their cross-section. Gratings with a period of $560 \mathrm{~nm}$ were subsequently bonded on the waveguides for input coupling. Thermal response was characterized by monitoring the coupling angle at different temperatures. Temperature dependences of the incident angle at two main peaks of $0.0027^{\circ} / \mathrm{K}$ and $0.0054^{\circ} / \mathrm{K}$ were determined respectively at the wavelength of $852 \mathrm{~nm}$ and a linear response over the evaluated range between $298 K$ and $323 K$ was observed, which opens up possible applications for on-chip temperature monitoring and thermal compensation when integrated with polymer sensors.
\end{abstract}

Keywords: Integrated optics devices, polymer optical sensors, polymer grating coupler, waveguides, low-cost mass-production

\section{INTRODUCTION}

Waveguide based polymer optical sensors have attracted significant scientific interest due to the advantages of flexibility, low cost and mass-production possibilities. ${ }^{1,2}$ The transfer from well-established inorganic materials to all polymer based sensors has a great potential to provide cost-effective disposable sensors. However, the main challenge in the integration of full functional all-polymer systems, which is interfacing waveguides based sensors to light sources, remains. Coupling strategies such as end-facet coupling, $45^{\circ}$ mirrors and gratings have been investigated to overcome this challenge. ${ }^{3-5}$ But facet polishing is complicated and difficult for large flexible foils, while $45^{\circ}$ mirrors often require a metal reflection layer. Furthermore, the fabrication costs needed for manufacturing $45^{\circ}$ mirrors remain high and thus it is not suitable for disposable sensors especially when only a single use is required. High efficient surface grating couplers have been well established for inorganic waveguide sensors ${ }^{6,7}$ however implementing polymer grating couplers is still under development due to the limited refractive index contrast of polymer materials. ${ }^{5}$ Furthermore, sub-wavelength grating is needed for the coupling feature

Further author information: (Send correspondence to Pei Li)

Pei Li: E-mail: pei.li@imtek.uni-freiburg.de, Telephone: +49 (0)761 2037568

Integrated Optics: Devices, Materials, and Technologies XXII, edited by Sonia M. García-Blanco,

Pavel Cheben, Proc. of SPIE Vol. 10535, 105351R · () 2018 SPIE

"CCC code: 0277-786X/18/\$18 - doi: 10.1117/12.2289444

Proc. of SPIE Vol. 10535 105351R-1 
and the period size of hundreds of nano-meters makes it as the major part of the fabrication costs of the optical sensors. Moreover, the single mode waveguide has small cross section, which causes small overlap region of the incoming light and grating coupler, and thus it is even more difficult to realize grating coupler for single mode waveguide than slab waveguide. All these factors limit the reversibly use of grating coupler for single mode waveguide based disposable sensors.

Besides, polymers have higher thermo-optic-coefficients and thermal expansion coefficients compared to inorganic materials, making them very sensitive to temperature changes. ${ }^{8}$ Consequently, polymer grating couplers show a shift of the coupling angle with temperature and thus a decay of efficiency.

We therefore present a temperature characterization of all-polymer based reusable grating coupler on waveguide. Cost-effective manufacturing methods, including hot-embossing and spin-coating, were used for the fabrication of waveguides and gratings on separate polymethyl methacrylate (PMMA) foils. Single mode waveguides were realized by modifying the dimensions of their cross-section. Gratings and tapers were optimized for near vertical coupling of the collimated light into the single mode waveguide. PMMA foil with hot-embossed grating structures was subsequently bonded on the waveguide foil via van der Waals force for input coupling, which allows separating the grating from the waveguide without damaging both foils afterwards. Thermal response was characterized by monitoring the coupling angle at different temperatures of the proposed reusable coupler. A temperature dependence of the incident angle of $0.0027^{\circ} / K$ and $0.0054^{\circ} / K$ for the two main peaks was determined for the laser beam with a wavelength of $852 \mathrm{~nm}$ and a linear response over the evaluated range between $298 K$ and $323 K$ was observed. The temperature response may open up possible applications for on-chip temperature monitoring and thermal compensation when integrated with polymer sensors.

\section{DESIGN}

Sub-wavelength grating is used to couple the collimated incident light beam into the waveguide, as shown in Fig. 1. A PMMA foil with structured binary grating is bonded on the sensor foil via van der Waals force, and collimated laser beam is coupled and converted into single mode waveguides by tapers.
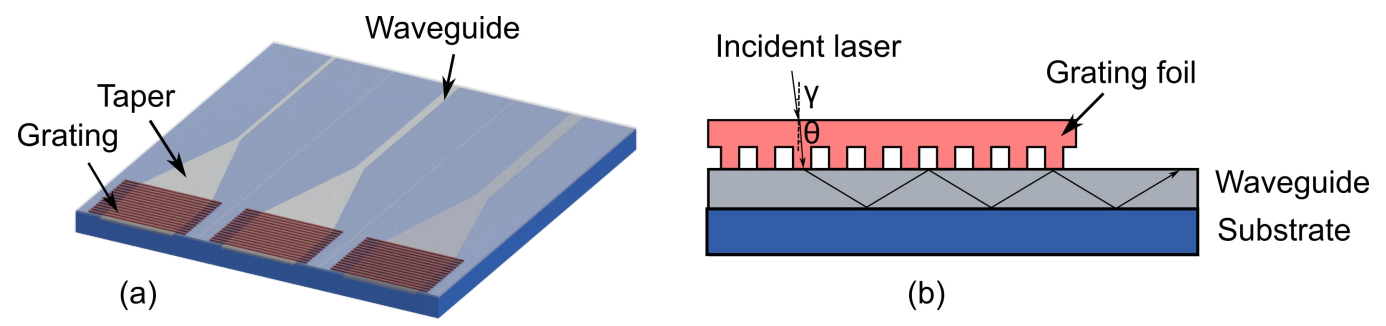

(b)

Figure 1. (a) Schematic diagram of the proposed waveguide coupling scheme via a sub-wavelength grating and taper. (b) Side view along with the wave propagation direction in the waveguide.

Inverted rib waveguide has been implemented in our sensors, due to its larger dimension of the cross section when compares to ridge waveguide, as shown in Fig. 2. The cross section with $\mu m$ range makes the fabrication of the master structure less critical and more comparable to roll-to-roll replication process. Single mode behavior can be modified by optimizing the overall core height, the width and depth of the rib groove. ${ }^{9}$ The optimized parameters in this design, are $3 \mu \mathrm{m}, 3.5 \mu \mathrm{m}$, and $0.6 \mu \mathrm{m}$ with height, width and depth respectively.

For coupling the collimated laser beam into waveguide, the period of the grating coupler, $\Lambda$, has to fulfill the wave vector matching condition:

$$
\frac{m \lambda}{\Lambda}=N_{e f f}-n_{c} \sin \theta,
$$

where $\lambda$ is the wavelength of the incident light source, $m$ is the coupled diffractive order of the grating, $N_{\text {eff }}$ is the effective refractive index of waveguide mode, $n_{c}$ is the refractive index of cladding layer, and $\theta$ is the angle 


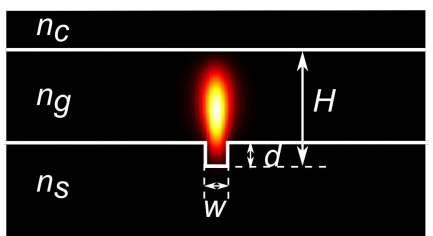

Figure 2. Cross-sectional view of an inverted rib waveguide: the intensity distribution of the fundamental mode is simulated by Photon Design.

of incidence in the cladding layer. As shown in Fig. 1b, the light source is fixed on an angular scanner in air and thus the incident angle in air $\gamma$ is measured. According to Snell's Law, the coupling angle $\gamma$ can be expressed as

$$
\sin \gamma=n_{c} \sin \theta=N_{e f f}-\frac{m \lambda}{\Lambda} .
$$

Since polymers have higher thermo-optic-coefficients (TOC) and thermal-expansion-coefficients (TEC) compared to inorganic materials, makes them more sensitive to temperature changes. Consequently, polymer grating couplers show a shift of the coupling angle with temperature and thus a decay of efficiency. The changes in coupling angle in air as a function of temperature changes can be modified in Eq. 3:

$$
\sin \gamma=N_{e f f}(1+\beta \Delta T)-\frac{m \lambda}{\Lambda(1+\alpha \Delta T)},
$$

where $\alpha$ is the positive thermal-expansion-coefficient of the grating, and $\beta$ is the negative thermo-opticcoecient of $N_{\text {eff }}$. Since the grating coupler is designed to work for the nearly vertical input, thus $\gamma \approx \sin \gamma$. The reciprocal term of Eq. 3 introduces a non-linearity into the sinusoidal function of $\gamma$, and its prominence is strongly dependent on the value of of the material. Moreover, the negative TOCs of the optical polymer materials are in the same order of magnitude as their positive TECs. And both thermal effects act against each other in Eq. 3, requiring a very careful selection and characterization of the used materials. Therefore polymer grating couplers show a signicantly higher complexity of the thermal behavior compared to silicon gratings.

\section{FABRICATION}

The waveguides and gratings were both fabricated on commercial PMMA foils (PLEXIGLAS) via hot-embossing method. The process of the waveguide manufacturing started with a fabrication of a silicon stamper which will be later used as a master structure during hot-embossing process. A $0.6 \mu m$-thick silicon nitride layer was firstly deposited on a polished silicon wafer via plasma-enhanced chemical vapor deposition (PECVD). Photolithgraphy was used to pattern the designed rid waveguides with tapers on the top of silicon nitride. And the deposited silicon nitride layer is subsequently etched by reactive ion etching (RIE) process. Vertical wall and homogenous etch depth have been achieved over the entire wafer. The height of the inverted rib grooves can be tuned by changing the thickness of the deposited silicon nitride layer, according to the design. Due to the required large area of the grating for increasing the coupling efficiency together with the sub-wavelength periodic structure, micro fabrication method such as electron-beam lithography is not suitable for the fabrication of the grating stamper. A commercial available grating stamper is thus used as a master in the hot-embossing process.

The patterns on both stampers were subsequently transferred on the surface of pre-heated PMMA foils by using a commercial hot embossing machine (HEX03, Jenoptik Mikrotechnik GmbH, Germany). Both stamper and PMMA foil were firstly heated up to the molding temperature of $140{ }^{\circ} \mathrm{C}$. After that the hard stamp was pressed into the PMMA foil with an embossing force varying from 500 to $4000 \mathrm{~N}$, which is determined by the thickness of the pre-patterned structure. In the end the stamper and PMMA foil were demolded at the temperature of $50{ }^{\circ} \mathrm{C}$.

The microscopic image of the hot-embossed PMMA foil with the waveguide and taper structures is shown in Fig. 3a, and the corresponding parameters of the designed structure are shown in Fig. 3b. A typical surface 
roughness of about $30 \mathrm{~nm}$ has been evaluated by white light interferometer characterization, which is comparable with the roughness of the silicon master wafer. The height of the embossed waveguide rib groove is $625.5 \pm 2.5$ $n m$, which is about $4 \%$ deeper than the design. The precision of the depth of the waveguide groove and the surface roughness can be further improved by maintaining the silicon nitride deposition process for the stamper.

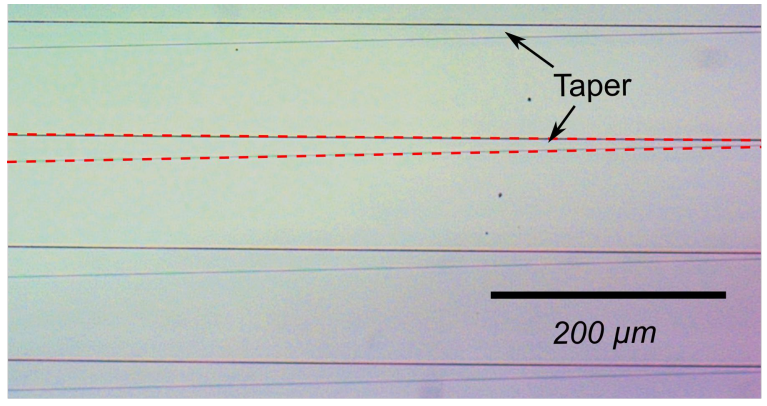

(a)

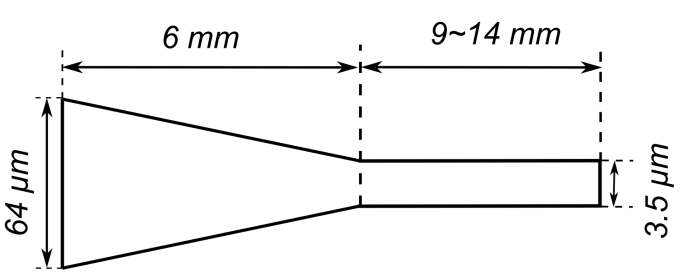

(b)

Figure 3. (a) Optical micrograph of taper arrays in a hot-embossed PMMA foil; (b) corresponding parameters of the designed structure.

Fig. 4 shows an atomic force micrograph (AFM) image of the replicated grating in PMMA foil. The overall transfer quality of the structures in PMMA was good, except that the top edges of the grating were rounded as expected for thermal process such as hot-embossing. The period and etch depth of grating were measured to be $560 \mathrm{~nm}$ and $150 \mathrm{~nm}$, which corresponds to the expected value from the stamper.

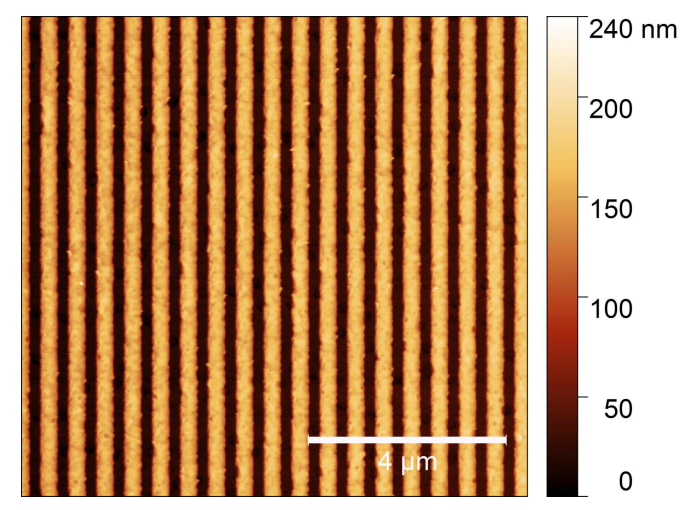

Figure 4. Atomic force micrograph of the replicated grating in PMMA foil.

The hot-embossed waveguide foil was afterwards spin-coated by a custom made polymer, UG45E ${ }^{10}$ to obtain a core layer with an inverted rib cross section. This UG45E consists of $55 w t$.\% Syntholux and $45 w t . \%$ ethylene glycol dimethacrylate (EGDMA). And Diphenyl (2,4,6-trimethylbenzoyl) phosphine oxide (DPO) was added as photo initiator. The layer thickness of the core material is controlled by the rotation speed of the spin-coating process and we achieved a layer thickness of $3 \mu \mathrm{m}$ with a speed of $3200 \mathrm{rpm}$. To evaluate the deposition quality of the core layer and later on observe the coupled mode on the end facet of the waveguide, the two facets of the waveguide foils were grinded and polished. Fig. 5(a) and 5(b) show optical images of the taper facet and single mode waveguide facet respectively. A concave profile was observed on the taper grooves, which causes the decrease of the total thickness of the core layer on the taper, as expected for spin-coating process on large grooves. While such influence can be neglected in the single mode waveguide case. This may cause a shift of the coupling angle due to the tuning of the effective refractive index of the taper. 


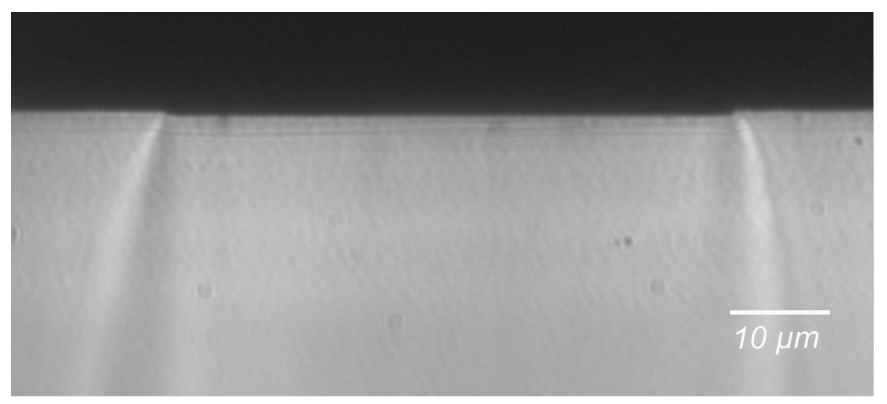

(a)

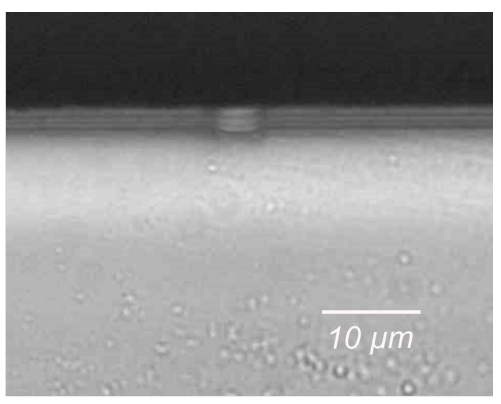

(b)

Figure 5. Optical microscope image of the polished waveguide facet: (a) at the taper side and (b) at the single mode waveguide side.

\section{THERMAL CHARACTERIZATION OF THE MATERIAL}

For the purpose of temperature characterization of the polymer grating couplers, the refractive indices of the PMMA substrate and the core material UG45E with changes of temperature were characterized by using a digital multi-wavelength refractometer DSR- $\lambda$ (Schmidt+Haensch, Germany). PMMA foil with a diameter of $8 \mathrm{~mm}$ was prepared to fit the size of the measurement chamber. Since free-standing thin layer of the core material UG45E is difficult to achieve, we used a molded cylinder with a dimeter of $8 \mathrm{~mm}$ and thickness of 4 $\mathrm{mm}$ for the measurement. The cylinder was prepared by a PDMS mold and cured under UV light. The samples were measured in a wavelength range from $404.7 \mathrm{~nm}$ to $706.5 \mathrm{~nm}$ and in a temperature range from $293 \mathrm{~K}$ to $323 \mathrm{~K}$ with a step of $10 \mathrm{~K}$. Since the refractive index value at $852 \mathrm{~nm}$ cannot be measured directly from the refractometer, exponential fitting was applied to the measurement results. Fig. 6 shows the refractive indices measurement results of UG45E and the fitted results. The refractive index decreases from 1.570 to 1.541 along with the wavelength change from $404.7 \mathrm{~nm}$ to $706.5 \mathrm{~nm}$ at $293 \mathrm{~K}$. The fitted refractive index value of PMMA and UG45E at $852 \mathrm{~nm}$ as a function of temperature is further plotted in Fig. 7(a). The TOC of PMMA foil and UG45E extracted from the linear fit are $-1.85 \times 10^{-4} K^{-1}$ and $-1.04 \times 10^{-4} K^{-1}$ respectively, which are in the same range of the reported value in the literature. ${ }^{11,12}$

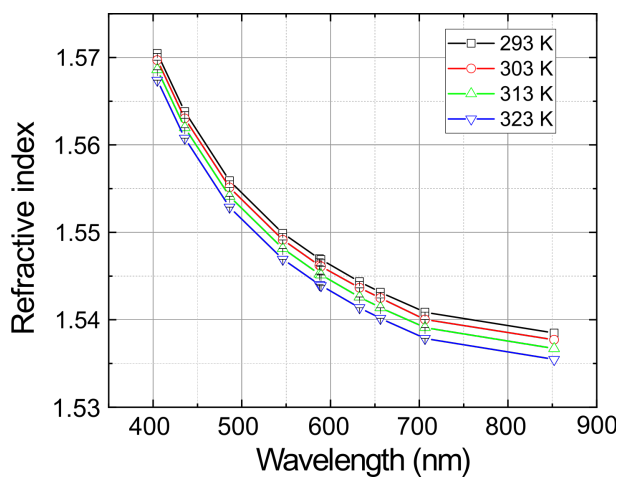

Figure 6. Refractive indices of the core material UG45E at different wavelength.

The refractive indices values of PMMA and UG45E plotted in Fig. 7(a) have been taken for the simulation of $N_{\text {eff }}$ of the fundamental mode of the taper by using Photon Design, and the results are shown in Fig. $7(\mathrm{~b})$. The TOC extracted from the linear fit of the waveguide structure is $-1.01 \times 10^{-4} \mathrm{~K}^{-1}$. These measurements and simulations give a starting point for the temperature characterization of the grating coupler, although the TOC of the bulk cylinder of UG45E used in the material characterization may deviate from the thin layer actually used for waveguide fabrication due to the different curing conditions. 


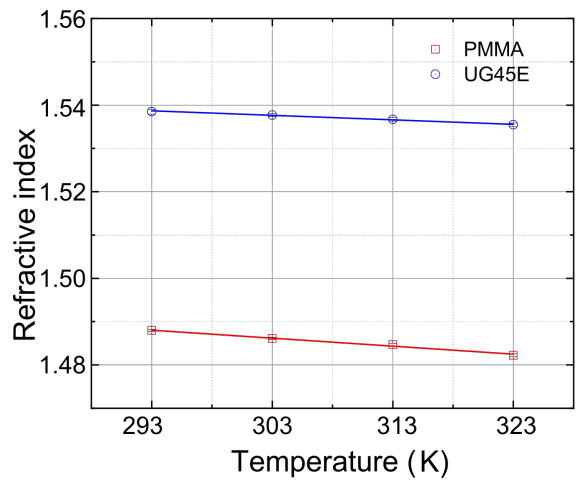

(a)

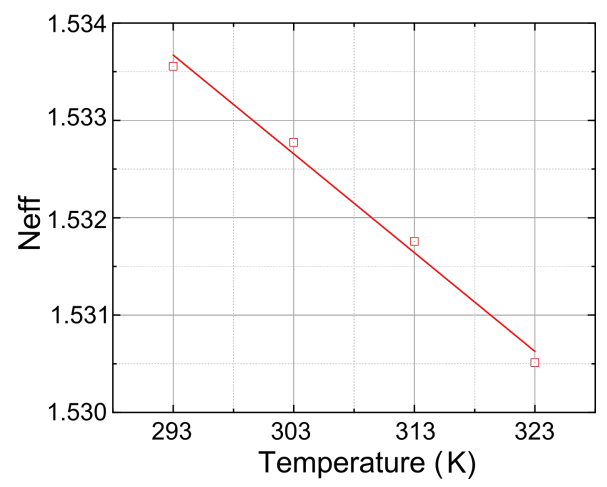

(b)

Figure 7. Refractive index as a function of the temperature: (a) fitted line slope for PMMA: $-1.85 \times 10^{-4} K^{-1}$ and UG45E: $-1.04 \times 10^{-4} K^{-1}$, and (b) fitted line slope for Neff:-1.01 $\times 10^{-4} K^{-1}$.

\section{MEASUREMENT}

The measurement setup for temperature dependence characterization of the coupler is shown in Fig. 8. The grating foil was reversibly bonded onto a waveguide foil van der Waals force. The bonded foils were then mounted onto a 3-axis precision stage and fixed by a $5 \mathrm{~mm}$ thick PMMA plate to avoid the movement of the facet. A Peltier temperature control element was attached to the 3-axis stage for temperature controlling. A close-loop temperature control was achieved by measuring the real-time temperature via a standard Pt100 resistive platinum temperature sensor and generating the controlling current by a Newport 3040 temperature controller.

A near infrared laser diode with a wavelength of $852 \mathrm{~nm}$ was connected to a collimator by single mode fiber. The collimator is mounted on a rotational stage with a resolution of $0.007^{\circ}$. The rotation center of the stage was aligned to the interface of the grating and the waveguide structure, applying scan of the incident angle of the laser beam without change the position of it. The intensity of the out coupled mode was capture by a microscope objective and imaged by a CMOS camera chip.

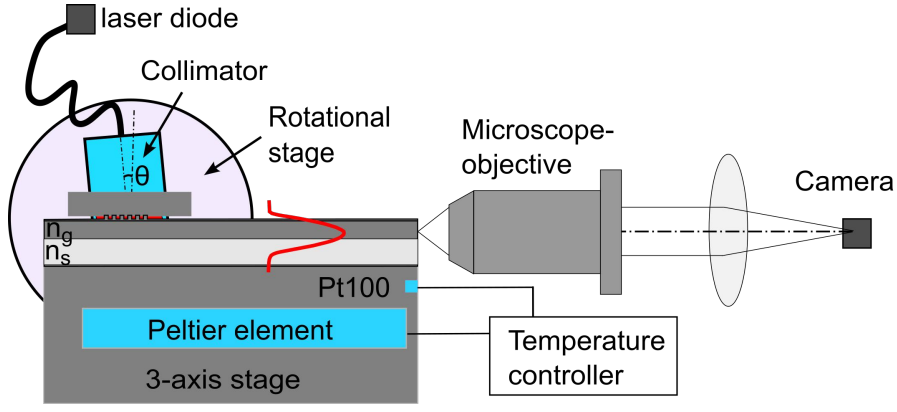

(a)

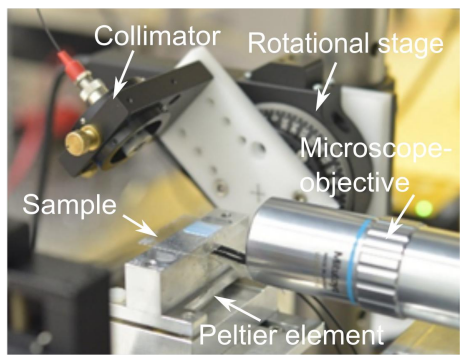

(b)

Figure 8. (a) Schematic image and (b) photo of the optical measurement set-up for temperature dependence characterization.

To characterize the temperature dependence of coupling angle of the proposed coupler, the intensity as a function of the incident angle in a temperature range from $298 \mathrm{~K}$ to $323 \mathrm{~K}$ was measured. This covers the main operation temperature range for our sensors. Lower temperature can be achieved by increasing the area of the Peltier temperature controller. However measurement at higher temperature is difficult to achieve due to the large vibration of facet caused by the thermo expansion. Fig. 9 shows an example of intensity response of a grating and taper coupled straight single mode waveguide at three different temperatures. Due to the taper 
behaviors as a multi-mode waveguide, light was coupled into higher modes of the taper at lower incident angles, which causes the sub-peaks at the left side of the main peak, as shown in inset in Fig. 9. These coupled mode were subsequently converted into the fundamental mode of the single mode waveguide following a scattering matrix. The intensity of the coupled light generally dropt along with the increase of the temperature, and the coupling angles of two central peaks were both increased towards large angles. The change of the angle at these two peaks over the temperature range from $298 K$ to $323 K$ is plotted in Fig. 10. Both results show good linearity over the measurement range and the fitted lines show $0.0027^{\circ} / K$ and $0.0054^{\circ} / K$ temperature dependence of the coupler respectively. The higher mode with a coupling angle around $-0.3^{\circ}$ is more sensitive to the temperature changes than the fundamental mode with a coupling angle around $0.6^{\circ}$. This feature can be exploited for a coarse on-chip integrated temperature monitoring and thermal compensation when integrated with polymer sensors, although the accuracy is limited to about $1 K$ with the provided angle scanning resolution.

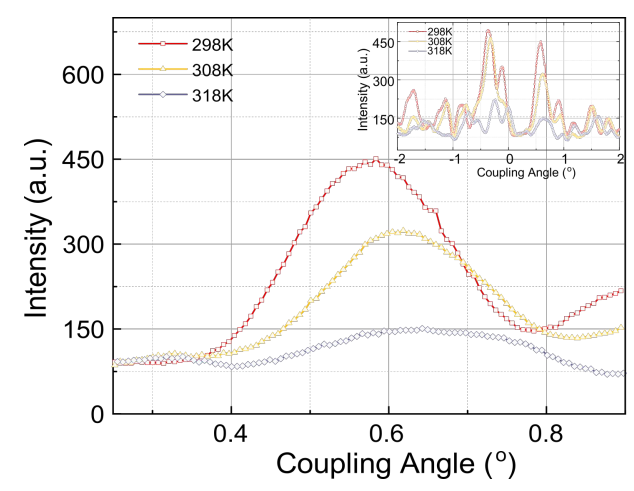

Figure 9. Experimental results of the out-put intensity as a function of angular detuning at different temperatures.

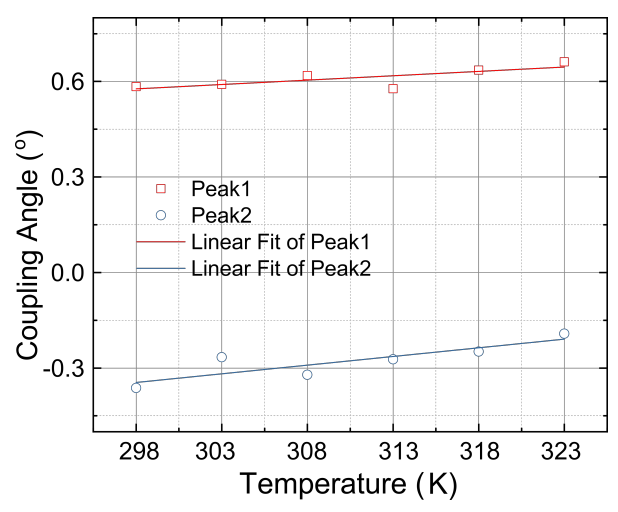

Figure 10. Temperature dependence of the coupling angle of two central peaks.

\section{CONCLUSION}

We introduced a temperature characterization of all-polymer based grating couplers on single mode waveguides. Gratings with a period of $560 \mathrm{~nm}$ were reversibly bonded on tapers for nearly vertical light coupling. Costeffective manufacturing methods, including hot-embossing and spin-coating, were used for the fabrication of waveguides and gratings on PMMA foils. Single mode waveguides were realized by modifying the dimensions of the inverted rib cross-section. Thermal response was characterized by monitoring the coupling angle at different temperatures. Temperature dependences of the incident angle at two main peaks of $0.0027^{\circ} / \mathrm{K}$ and $0.0054^{\circ} / \mathrm{K}$ were determined at the wavelength of $852 \mathrm{~nm}$ and linear responses over the evaluated range between $298 \mathrm{~K}$ and $323 K$ were observed, which opens up possible applications for on-chip temperature monitoring and thermal compensation when integrated with polymer sensors. 


\section{REFERENCES}

[1] Rigo, E., Aparicio, F., Vanacharla, M., Larcheri, S., Guider, R., Han, B., Pucker, G., and Pavesi, L., "Evanescent-field excitation and collection approach for waveguide based photonic luminescent biosensors," Applied Physics B 114(4), 537-544 (2014).

[2] Kozma, P., Kehl, F., Ehrentreich-Frster, E., Stamm, C., and Bier, F. F., "Integrated planar optical waveguide interferometer biosensors: A comparative review," Biosensors and Bioelectronics 58, 287-307 (2014).

[3] Morimoto, Y. and Ishigure, T., "Low-loss light coupling with graded-index core polymer optical waveguides via 45-degree mirrors," Optics express 24(4), 3550-3561 Optical Society of America.

[4] Bruck, R., Muellner, P., Kataeva, N., Koeck, A., Trassl, S., Rinnerbauer, V., Schmidegg, K., and Hainberger, R., "Flexible thin-film polymer waveguides fabricated in an industrial roll-to-roll process," Applied optics 52(19), 4510-4514 (2013).

[5] Prokop, C., Schmalz, T., Kleessen, P., Laegel, B., Wolff, S., Mitchell, A., and Karnutsch, C., "Tunable air-suspended polymer grating couplers," Optical Engineering 56(6), 067112-067112 (2017).

[6] Taillaert, D., Bogaerts, W., Bienstman, P., Krauss, T. F., Van Daele, P., Moerman, I., Verstuyft, S., De Mesel, K., and Baets, R., "An out-of-plane grating coupler for efficient butt-coupling between compact planar waveguides and single-mode fibers," IEEE Journal of Quantum Electronics 38(7), 949-955 (2002).

[7] Li, H., Zhou, W., Zhang, M., Liu, Y., Zhang, C., Li, E., Miao, C., and Tang, C., "Large-area binary blazed grating coupler between nanophotonic waveguide and led," The Scientific World Journal 2014 (2014).

[8] Bhuvaneshwaran, A., Sherman, S., and Zappe, H., "Spectral response of bragg gratings in multimode polymer waveguides," Applied Optics 56(34), 9573-9582 Optical Society of America.

[9] Hofmann, M., Xiao, Y., Sherman, S., Gleissner, U., Schmidt, T., and Zappe, H., "Asymmetric machzehnder interferometers without an interaction window in polymer foils for refractive index sensing," Applied optics 55(5), 1124-1131 (2016). Optical Society of America.

[10] Gleiner, U., Khatri, B., Megnin, C., Sherman, S., Xiao, Y., Hofmann, M., Gnther, A., Rahlves, M., Roth, B., and Zappe, H., "Optically and rheologically tailored polymers for applications in integrated optics," Sensors and Actuators A: Physical 241, 224-230 Elsevier.

[11] Zhang, Z., Zhao, P., Lin, P., and Sun, F., "Thermo-optic coefficients of polymers for optical waveguide applications," Polymer 47(14), 4893-4896 (2006).

[12] Weber, M. J., [Handbook of optical materials], vol. 19, CRC press (2002). 